\title{
Archéopages
}

Archéopages

Archéologie et société

34 | 07/2012

Campagnes

\section{Relevé en 3D d'une structure souterraine médiévale}

Chhavy-Cyril Tan et Laurent Fournier

\section{(2) OpenEdition}

1 Journals

Édition électronique

URL : https://journals.openedition.org/archeopages/424

DOI : 10.4000/archeopages.424

ISSN : 2269-9872

Éditeur

INRAP - Institut national de recherches archéologiques préventives

Édition imprimée

Date de publication : 1 février 2012

Pagination : 99-101

ISSN : 1622-8545

\section{Référence électronique}

Chhavy-Cyril Tan et Laurent Fournier, «Relevé en 3D d'une structure souterraine médiévale »,

Archéopages [En ligne], 34 | 07/2012, mis en ligne le 01 juillet 2012, consulté le 24 janvier 2022. URL: http://journals.openedition.org/archeopages/424 ; DOI : https://doi.org/10.4000/archeopages.424

(C) Inrap 
confirmer ou infirmer des hypothèses de contenants et réaliser des illustrations [ill. 2]. De plus, tous les modèles peuvent être fournis aux différents acteurs de l'étude au format pdf $3 \mathrm{D}$, facilement lisible avec Adobe Reader ou Acrobat [ill. 3], qui permet de visionner les acquisitions $3 \mathrm{D}$ à l'échelle, de faire des mesures et de pratiquer des coupes. La photogrammétrie a ici permis de trouver une nouvelle approche d'étude des incinérations. Différentes hypothèses de formes de contenant peuvent être confrontées à une représentation virtuelle, afin de les valider - ou non - et de les illustrer. De plus, la forme et les couleurs de faits archéologiques, qui seront à terme démantelés par la fouille, peuvent être sauvegardées. Enfin, cet enregistrement peut alimenter une base de données utile pour des études ultérieures.

\section{Modélisation de terrain par prise de vue embarquée}

Régis Touquet, Inrap

Une campagne de prise de vues aériennes par drone a été réalisée sur l'opération archéologique de Morigny-Champigny (Essonne), couvrant $5000 \mathrm{~m}^{2}$ et divisée en trois zones distinctes. La couverture photographique complète de l'emprise et l'importante quantité d'images produites par un tel procédé a permis d'exploiter les clichés dans un travail de modélisation de leur géométrie. Il est en effet possible, grâce à la technique de la photogrammétrie, d'exploiter des images diverses tant pour leur échelle que par les types d'appareils de prises de vues. Outre la possibilité de mettre à l'épreuve cette technique sur une grande surface, le but était de créer un modèle numérique de terrain précis des différentes zones de fouilles et ce, uniquement à partir des documents photographiques. La société Kyuaero a réalisé les prises de vues aériennes à l'aide d'un drone commandé depuis le sol. Il s'agissait d'obtenir des photographies zénithales ainsi que des photographies angulaires, le drone survolant alors de manière concentrique les emprises à traiter. Les clichés ont été pris en mode rafale, à environ 20 mètres du sol, à raison d'une photographie par seconde. Le couvert nuageux entrainant une faible lumière le jour des prises de vue, la multiplication des images a ainsi été privilégiée. Un fond photographique d'une centaine de clichés exploitables par zones a été obtenu. Ces données raster brutes, d'une définition de 12 millions de pixels, ont ensuite été soumises à des solutions logicielles. Chaque cliché réalisé sur les zones de l'emprise a été traité de manière indépendante par les logiciels libres Bundler, PMVS, CMVS et Meshlab, afin d'en acquérir un modèle $3 \mathrm{D}$ à haute densité de points. Trois Modèles Numériques de Terrain (MNT) ont par la suite été calculés, couvrant des surfaces au sol de 600,1900 et $2500 \mathrm{~m}^{2}$. Un MNT se représente par des faces triangulaires modélisées en $3 \mathrm{D}$, qui traduisent le relief calculé. Le nombre de ces faces dépend davantage de la pertinence géométrale des sources de données utilisées que de la surface du modèle. Les logiciels d'acquisition $3 \mathrm{D}$ précités peuvent générer, selon la pertinence des informations, plusieurs millions de faces, offrant ainsi une visualisation très précise et fidèle des sujets traités. Le modèle obtenu doit être orienté selon le système de projection de l'opération. En amont du traitement, des points remarquables sont ainsi levés sur le terrain et déterminés par coordonnées. Ces données correspondent à des points d'angles ou d'arêtes identifiables sur le MNT calculé. Le géo-référencement du modèle en projection et en élévation permet ainsi d'analyser, de manière précise, la topographie existante et de la soumettre aux différents outils de visualisation. Des cartes en relief [ill. 1] des trois zones ont été dressées, facilitant ainsi la lecture topographique et la comparaison des altitudes d'une zone à l'autre. De plus, durant la phase de fouille et de post-fouille, un nombre infini de profils peuvent être exportés du modèle [ill. 2], traduisant graphiquement en $2 \mathrm{D}$ les informations du relief. Ces profils de terrains permettent également un contrôle de précision du MNT en le comparant à un levé traditionnel effectué par le topographe [ill. 3]. Toutefois, la résolution obtenue par la photogrammétrie s'avère beaucoup plus pertinente, car elle génère un nombre de points topographiques important, trop lourd à mettre en œuvre avec un levé au tachéomètre.

La confrontation des différentes couches superposables que sont le MNT, le plan-masse du site et les images géo-référencées issues de la photogrammétrie facilite la lecture des zones modélisées et permet une approche tridimensionnelle des problématiques. L'analyse des pentes, l'étude du microrelief, le calcul des cubatures font partie des outils utiles au responsable d'opération.

La procédure d'acquisition étant simple à mettre en œuvre, elle peut être répétée et permettre ainsi la comparaison précise de différents modèles successifs. Les niveaux d'occupation ou de décapage peuvent alors être documentés au fil de l'opération archéologique. La caractérisation des aspects du relief peut devenir systématique et intégrer une réflexion plus large sur l'implantation des occupations humaines.

\section{Relevé en 3D d'une structure souterraine} médiévale

Chhavy-Cyril Tan, Service archéologique départemental d'Indre-et-Loire Laurent Fournier, Inrap

La découverte, au cours de l'été 2009, d'une structure souterraine, annexe d'un habitat médiéval, sur le site d'Ormes « Le Bois d'Ormes » (Loiret) (Fournier, 2011) a conduit à la mise en 

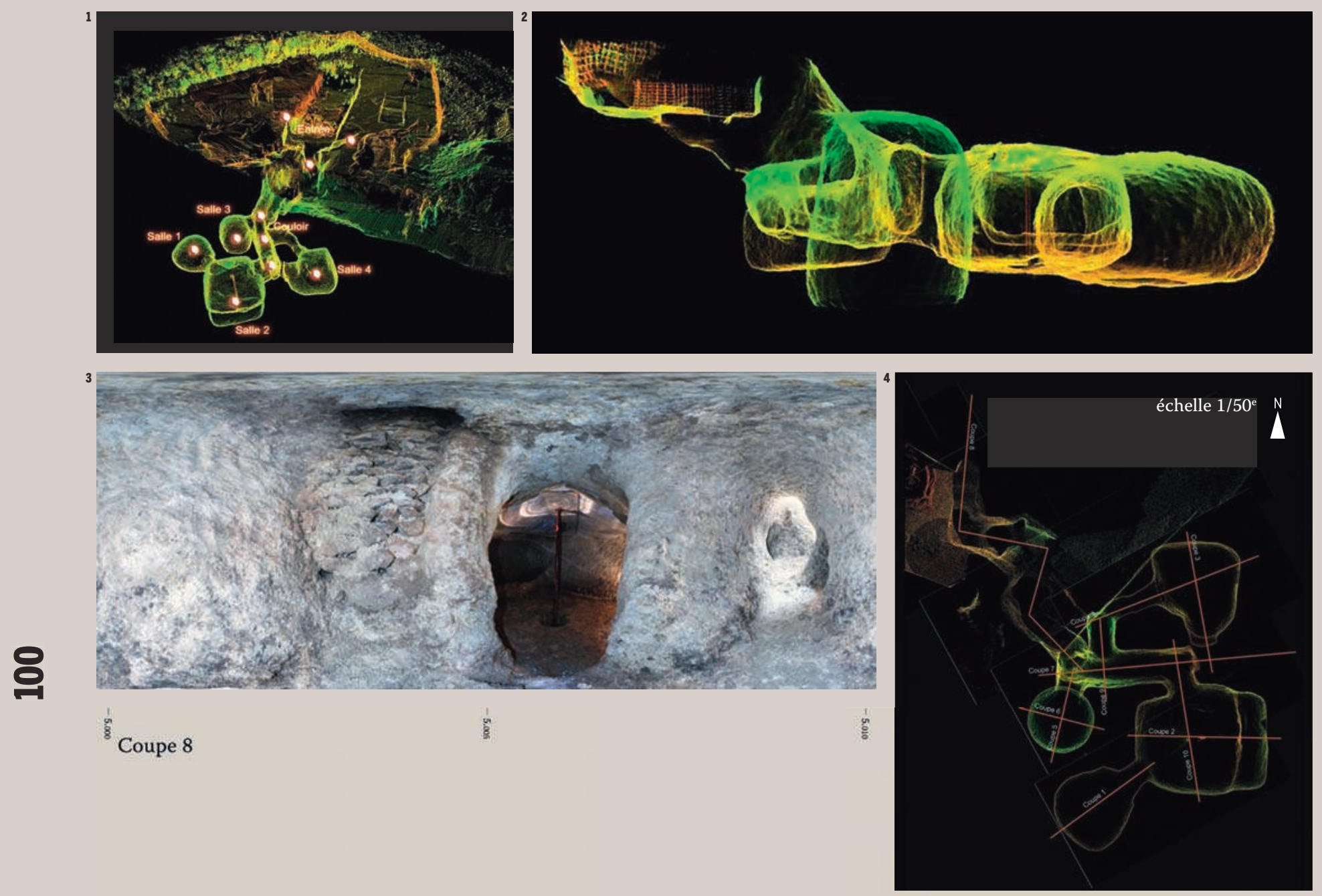

5

105

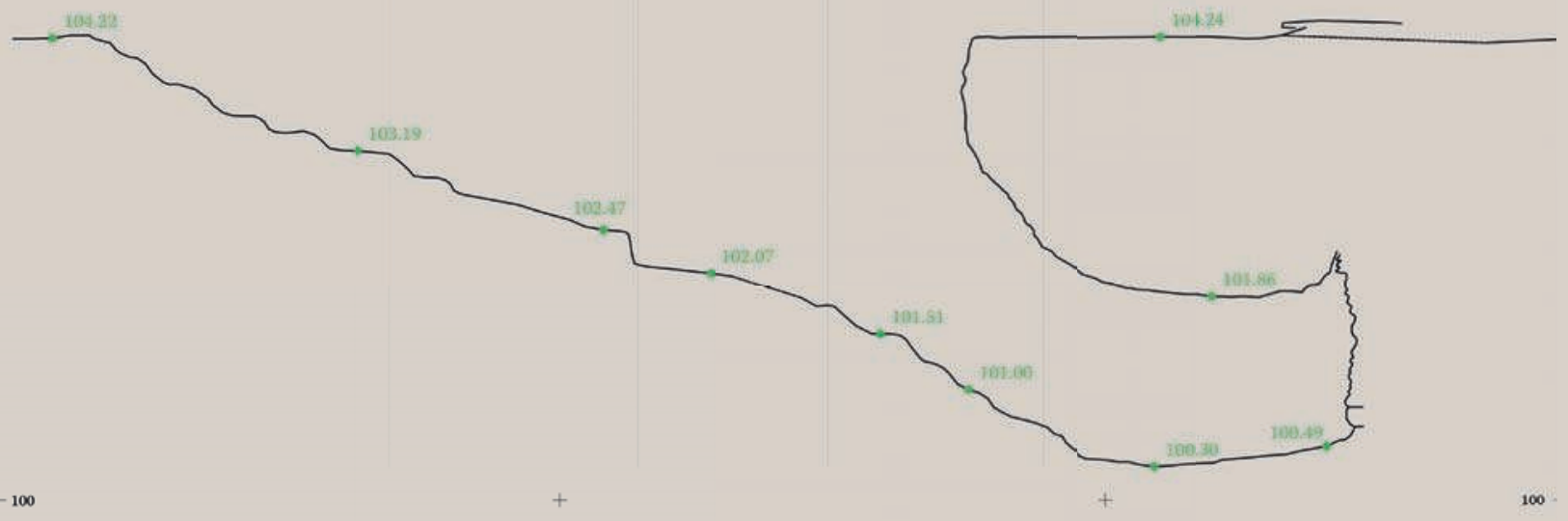

8ิ

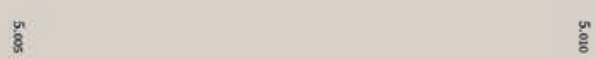

\begin{tabular}{|c|c|}
\hline $\begin{array}{l}\text { 1. Vue générale oblique du } \\
\text { souterrain et de la zone en } 30 \text {. } \\
\text { 2. Vue des profils cumulés } \\
\text { à } 360^{\circ} \text { de l'ensemble de } \\
\text { la structure souterraine. } \\
\text { 3. Prise de vue à } 360^{\circ} \\
\text { de l'extrémité sud-est } \\
\text { de la galerie principale }\end{array}$ & $\begin{array}{l}\text { "Le Bois d'Ormes ॥. } \\
\text { 4. Vue générale en plan } \\
\text { du souterrain et de la zone } \\
\text { en 3D avec implantation } \\
\text { des coupes et profills réalisés. } \\
\text { 5. Profil en long de l'ensemble } \\
\text { accès/galerie principale } \\
\text { du souterrain d'Ormes } \\
\text { "Le Bois d'Ormes » (coupe 8). }\end{array}$ \\
\hline
\end{tabular}

Système altimétrique local

Références bibliographiques

FourniER L. (DIR.), 2011, Ormes, «Le Bois d'Ormes » (Loiret), Rapport final d'opération, Inrap, SRA Centre, Orléans, $707 \mathrm{p}$.

GILOTTE S. (DIR.), 2008, Communes d'Aschères-le-Marché et Villereau (Loiret), «Le Marjolt » et «La Cabane », site A19-C-8 ( $\left.n^{\circ} 45.009 .023 . A H\right)$, Rapport final d'opération, Inrap, SRA Centre, Orléans, p. 214-317. 
œuvre d'un relevé en $3 \mathrm{D}^{\mathbf{1}}$. Des relevés de ce type avaient été précédemment réalisés en région Centre, au cours des travaux préalables à la construction de l'autoroute A19, pour une structure similaire découverte sur le site de Villerau « Le Marjolet » (Loiret), (Gilotte et al., 2008, p. 272-317, fig. 126-128). Cependant, le rendu général en « fil de fer », les relevés manuels et les restitutions produites nous paraissaient insuffisants pour appréhender l'organisation générale de la structure et rendre compte des volumes des différentes galeries et cavités. Compte tenu du laps de temps relativement réduit dédié à cette partie de lintervention (dix jours ouvrés), nous avons opté pour une méthode de relevé plus rapide et plus complète en faisant appel à la société de géomètres experts « Axis conseil ».

La fouille et le relevé classique des stratigraphies et des profils des principales cavités et galeries de ce souterrain ont été réalisés en sept jours. À l'issue de cette première phase d'intervention, trois journées ont été nécessaires pour la réalisation du relevé des contours du souterrain à l'aide d'un émetteur laser permettant de modéliser les volumes et de procéder à une photogrammétrie ${ }^{2}$ et quelques jours supplémentaires ont permis l'exploitation des données et l'élaboration des plans, coupes et profils.

Le premier essai de modélisation a consisté en une tentative de texturation numérique (morphing), qui n'était pas satisfaisante car trop illisible [ill. 1-2]. Puis nous ont été fournies des vues à $360^{\circ}$ des différentes composantes du souterrain, intéressantes malgré quelques déformations, mais qui restent difficiles à exploiter [ill. 3]. La dernière étape a été un mélange entre traitement $3 \mathrm{D}$ et photogrammétrie, avec l'application des prises de vue dans la modélisation $3 \mathrm{D}$ [ill. 4].

Les apports de ce type de relevé peuvent être exposés en quelques points. Le premier est la rapidité d'acquisition des données car, pour ce type de structure, le recours à un relevé classique s'avère difficile à réaliser sans procéder à l'ouverture des voûtes. Ces terrassements, outre le fait qu'ils entrainent la destruction d'une grande partie du terrain sondé, peuvent également fragiliser la construction et entrainer la condamnation de tout ou partie de l'ouvrage. Deuxièmement, la précision des prises de points réduit la marge d'erreur par rapport à un traitement manuel, en particulier dans les endroits difficiles d'accès (pour traiter par exemple des traces d'outils). Troisièmement, la géolocalisation du relevé $3 \mathrm{D}$ annule les divers problèmes d'orientation inhérents à toutes les structures souterraines. L'ultime argument en faveur de cette méthode est la facilité de manipulation et d'interprétation de la structure. Il a suffi de demander une vue verticale générale et de choisir les différents profils voulus, en particulier le profil du développé général du souterrain [ill. 5].

Le recours à la restitution en $3 \mathrm{D}$ pour le souterrain d'Ormes permet un certain nombre de réflexions autour du traitement des cavités souterraines. Une étude préliminaire apparaît indispensable tant du point de vue de la sécurité des différents intervenants, que du point de vue logistique. Cela permet en effet une organisation de la fouille visant à favoriser la mise en place d'une méthodologie associée à l'emploi de ce type de technologie. Contrairement à l'idée encore trop répandue dans la communauté archéologique, la modélisation en $3 \mathrm{D}$ ne sert pas uniquement à la valorisation des vestiges ${ }^{3}$ : elle permet également un enregistrement rapide et précis, et offre une possibilité de traitement a posteriori de l'ensemble des données acquises.

\section{La maquette numérique issue de levés $3 \mathrm{D}$}

Sylvie Eusèbe, Inrap

Le relevé total de vestiges semble désormais accessible grâce à la maquette virtuelle en $3 \mathrm{D}$, issue de la photogrammétrie numérique ou du laser-scanner, procédés qui captent mécaniquement la réalité dans sa géométrie et ses couleurs. Considérée comme un summum de justesse, d'exhaustivité et d'objectivité, cette maquette numérique est souvent présentée comme le support idéal, quelles que soient les études envisagées, à la différence d'un relevé traditionnel spécifique au secteur de l'étude à laquelle il participe. Il convient néanmoins de ne pas oublier qu'un modèle numérique est dit objectif en raison de l'absence d'observation et d'interprétation directement associées, et qu'il ne constitue pas, à lui seul, un « relevé archéologique».

En archéologie préventive, ces technologies mises en œuvre dans un cadre expérimental devraient permettre, entre autres, de tester les différentes méthodes de modélisation et la qualité (précision, résolution, etc.) des résultats selon des protocoles connus et décrits. Elles devraient surtout nous inviter à nous interroger sur nos besoins actuels et à venir en termes de précision nécessaire et suffisante pour tel ou tel type d'observation, ainsi que, plus généralement, sur l'usage que les archéologues peuvent en faire.

La maquette numérique issue de levés en $3 \mathrm{D}$ renouvelle l'apport des vues perspectives et de la maquette " réelle », depuis longtemps utilisées en archéologie, comme outil d'aide à la recherche et comme support de communication. Cependant, de nouveaux défis devront être relevés pour assurer le succès de ces modèles numériques, notamment: garantir leur pérennité (indexation, archivage, métadonnées, interopérabilité) ; garantir leur qualité scientifique, ou tout au moins la connaitre; permettre leur appropriation par l'archéologue, car les objectifs scientifiques induisent les relevés à réaliser et les moyens à mettre en œuvre. La véritable innovation ne consiste ainsi plus à acquérir les compétences techniques pour réaliser ces modèles, mais plutôt, d'une part, à acquérir la maitrise de l'évolution qualitative des données produites et, d'autre part, à maitriser leur exploitation et leur présentation.
1. Nous renvoyons aux
débats et aux actes des

Journées informatique

sciencesconf.org/..

est une technique qui

permet de reconstitue

à partir d'une série

de photographies prises

selon un protocole adapte.

être envisagée avec la

d'animations vectorielles. 